OPEN ACCESS

Edited by:

Marino M. Bianchin,

Federal University of Rio Grande Do

Sul, Brazil

Reviewed by:

Jacopo Lanzone

Policlinico Universitario Campus

Bio-Medico, Italy

Daichi Sone,

University College London,

United Kingdom

*Correspondence:

Xing Fan

xingkongyaoxiang@163.com

Yinyan Wang

tiantanyinyan@126.com

Zhong Zhang

zhangzhong0502@163.com

Tao Jiang

taojiang1964@163.com

Specialty section

This article was submitted to

Epilepsy,

a section of the journal

Frontiers in Neurology

Received: 18 September 2020

Accepted: 12 February 2021

Published: 18 March 2021

Citation:

Fang S, Zhou C, Wang L, Fan X, Wang $Y$, Zhang $Z$ and Jiang $T$ (2021) Characteristic Alterations of Network

in Patients With Intraoperative

Stimulation-Induced Seizures During

Awake Craniotomy

Front. Neurol. 12:602716.

doi: 10.3389/fneur.2021.602716

\section{Characteristic Alterations of Network in Patients With Intraoperative Stimulation-Induced Seizures During Awake Craniotomy}

\author{
Shengyu Fang ${ }^{1,2}$, Chunyao Zhou ${ }^{1}$, Lei Wang ${ }^{1}$, Xing Fan ${ }^{1,2 *}$, Yinyan Wang ${ }^{1 *}$, Zhong Zhang ${ }^{1 *}$ \\ and Tao Jiang ${ }^{1,2,3 *}$ \\ 1 Department of Neurosurgery, Beijing Tiantan Hospital, Capital Medical University, Beijing, China, ${ }^{2}$ Beijing Neurosurgical \\ Institute, Capital Medical University, Beijing, China, ${ }^{3}$ Research Unit of Accurate Diagnosis, Treatment, and Translational \\ Medicine of Brain Tumors Chinese (2019RU11), Chinese Academy of Medical Sciences, Beijing, China
}

Background: The use of electrocorticography (ECOG) to avoid intraoperative stimulation-induced seizure (ISS) during awake craniotomy is controversial. Although a standard direct cortical stimulating (DCS) protocol is used to identify the eloquent cortices and subcortical structures, ISS still occurs. Epilepsy is related to alterations in brain networks. In this study, we investigated specific alterations in brain networks in patients with ISS.

Methods: Twenty-seven patients with glioma were enrolled and categorized into the ISS and non-ISS groups based on their history of ISS occurrence. A standard DCS protocol was used during awake craniotomy without ECoG supervision. Graph theoretical measurement was used to analyze resting-state functional magnetic resonance imaging data to quantitatively reveal alterations in the functional networks.

Results: In the sensorimotor networks, the glioma significantly decreased the functional connectivity (FC) of four edges in the ISS group, which were conversely increased in the non-ISS group after multiple corrections $(p<0.001$, threshold of $p$-value $=0.002$ ) Regarding the topological properties, the sensorimotor network of all participants was classified as a small-world network. Glioma significantly increased global efficiency, nodal efficiency, and the sigma value, as well as decreased the shortest path length in the ISS group compared with the non-ISS group ( $p<0.05)$.

Conclusions: The specific alterations indicating patient susceptibility to ISS during DCS increased global and nodal efficiencies and decreased the shortest path length and FC induced by gliomas. If the patient has these specific alterations, ECoG is recommended to monitor after-discharge current during DCS to avoid ISS.

Keywords: intraoperative stimulation-induced epilepsy, direct cortical stimulating, glioma, seizure, sensorimotor network 


\section{INTRODUCTION}

Awake craniotomy (AC) is the optimal approach to prevent neurological deficits when eloquent structures are invaded (1). Intraoperative stimulation-induced seizure (ISS) is the most serious complication of AC. Despite the application of a standard protocol of direct cortical stimulation (DCS) and using ice-cold Ringer's solution for control $(2,3)$, ISS is difficult to prevent (incidence was 2.2-21.5\%) (1,4-14). If ISS occurs, patients will have poor cooperation, and the time of functional monitoring will be prolonged, and the accuracy of identifying functional areas will be decreased. Accordingly, ISS prevention is crucial in $\mathrm{AC}$.

Electrocorticography (ECoG) is a minimally invasive technique of intraoperative neuro-monitoring. Whether or not ECoG should be used in AC to prevent ISS remains controversial $(2,4)$. Some traditional studies supported the use of ECoG during AC to establish the threshold of stimulation and capture after-discharges (15). In contrast, Boetto et al. (16) suggested that ECoG was unnecessary for AC. They proposed that ISS incidence was only $3.5 \%$ and that using ECoG would only complicate the surgical process. Hence, identifying patients susceptible to ISS is important to clarify the standards for using ECoG.

Seizures are considered related to brain network alterations. However, the association between ISS and alterations in functional networks remains poorly understood. Resting-state functional magnetic resonance imaging (rs-fMRI) with graph theoretical analyses can reveal the distribution of brain networks and identify changes in topological properties. Hence, we enrolled glioma patients who underwent AC and performed rsfMRI analysis using a graph theoretical approach. We aimed to find characteristic differences in functional networks between patients with and without ISS and to ascertain which patients are suitable for ECoG to capture after-discharges during AC.

\section{MATERIALS AND METHODS}

The institutional review board of Beijing Tiantan Hospital approved this study. All enrolled patients and participants provided written informed consent.

\section{Participants}

We retrospectively reviewed 84 patients diagnosed with gliomas who underwent AC with DCS to preserve motor, sensory, and motor-related language functions between March 2017 and March 2019 at Beijing Tiantan hospital. The inclusion criteria were as follows: (a) patient aged $>18$ years; (b) no history of biopsy, radiotherapy, or chemotherapy. The exclusion criteria were as follows: (a) contraindications for MRI; (b) head motion $>1 \mathrm{~mm}$ in translation or $1^{\circ}$ in rotation; and (c) the administration of antiepileptic drugs before preoperative rs-fMRI scanning. All enrolled patients were classified into epileptic (ISS) and non-epileptic (non-ISS) groups based on ISS occurrence. Moreover, all patients received $0.5 \mathrm{~g}$ levetiracetam twice a day to prevent glioma-related epilepsy after rs-fMRI scanning. We also recruited 20 healthy participants matched for age, sex, and education level.

\section{Clinical Characteristics}

Data for age, sex, education level, preoperative epilepsy, Karnofsky Performance Scale (KPS) score, and histopathology were derived from inpatient records and results of preoperative electroencephalograms. ISS and stimulation current information was derived from surgical records and intraoperative photos.

\section{Intraoperative Stimulation Protocol}

The stimulation protocol was the same as that described previously (17). The Ojemann stimulators with 5-mm diameter were employed to identify the eloquent cortices (intensity, 1$6 \mathrm{~mA}$; frequency, $60 \mathrm{~Hz}$; square wave). The stimulation current began at $1 \mathrm{~mA}$ and was increased by $0.5-\mathrm{mA}$ increments until the stimulation threshold was established. The stimulation threshold was determined by inducing unconscious movements (precentral gyrus stimulation) or transient numbness (postcentral gyrus stimulation). If the stimulation threshold were established, the stimulation current would remain constant and would be used to identify the eloquent cortices and subcortical structures. The duration of stimulation for identification was $1 \mathrm{~s}$ for sensorimotor-related structures and $4 \mathrm{~s}$ for language-related structures. No site was continuously stimulated. Whenever ISS occurred, ice-cold Ringer's solution was used to terminate it. If the seizure duration was over $10 \mathrm{~s}$, benzodiazepine medications were administered, and functional monitoring was discontinued. ECoG was not used to capture after-discharges or determine the stimulation threshold.

\section{MRI Acquisition}

A MAGNETOM Prisma 3T MR scanner (Siemens, Erlangen, Germany) was used to acquire all image data. The parameters of MRI sequences (T1, FLAIR, and rs-fMRI) were as follows: T1 magnetization-prepared rapid acquisition gradient echo with gadolinium enhancement to acquire anatomical images, repetition time (TR): 2,300 ms; echo time (TE): $2.3 \mathrm{~ms}$; field of view (FOV): $240 \times 240 \mathrm{~mm}^{2}$; flip angle: $8^{\circ}$; slice number: 192 ; voxel size: $1.0 \mathrm{~mm}^{3} \times 1.0 \mathrm{~mm}^{3} \times 1.0 \mathrm{~mm}^{3}$; FLAIR sequence, TR: 5,000 ms; TE: $387 \mathrm{~ms}$; FOV: $220 \mathrm{~mm}^{2} \times 220 \mathrm{~mm}^{2}$; flip angle: $150^{\circ}$; slice number: 128 ; thickness: $0.9 \mathrm{~mm}$; voxel size in panel: 0.4 $\mathrm{mm}^{3} \times 0.4 \mathrm{~mm}^{3} \times 0.9 \mathrm{~mm}^{3}$; rs-fMRI sequence, TR: $2,000 \mathrm{~ms}$; TE: $30 \mathrm{~ms}$; FOV: $220 \mathrm{~mm}^{2} \times 220 \mathrm{~mm}^{2}$; flip angle: $90^{\circ}$; slice number: 30 ; voxel size in panel: $3.0 \mathrm{~mm}^{3} \times 3.0 \mathrm{~mm}^{3} \times 3.0 \mathrm{~mm}^{3}$, acquisition duration: $8 \mathrm{~min}$.

Participants were asked to close their eyes without thinking about anything in particular during rs-fMRI acquisition.

\section{Regions of Tumor Invasion}

The MRIcron software (http://www.mccauslandcenter.sc.edu/ mricro/mricron/) was used to manually draw the extent of glioma invasion (shown in Supplementary Figure 1), in the individual patient images, by two neuroradiologists (with 10 years' experiences of glioma diagnosis) independently based on the enhanced regions of the FLAIR images for low-grade glioma and T1 enhancement images for high-grade glioma. If there was over $5 \%$ difference in the region between the two drawn images, a third neuroradiologist with 20 years' experience made the final decision. The drawn regions of tumor invasion of all patients 
were normalized into the MNI-152 T1 template using SPM 8 (University College London, London, United Kingdom; http:// www.fil.ion.ucl.ac.uk/spm/). The tumor volume was calculated using MRIcron software.

\section{Functional MRI Preprocessing}

A graph theoretical network analysis toolbox (GRETNA, https:// www.nitrc.org/projects/gretna) $(18,19)$ was used for rs-fMRI preprocessing. The pipeline of preprocessing was as follows (20): (a) data transformation (from DICOM to NIFTI); (b) removal of the first 10 images; (c) timing slice; (d) realignment; (e) normalization (normalized to the EPI template) (21); (f) smoothing (full width half maximum: $6 \mathrm{~mm}$ ); (g) linear detrending; (h) regressing out covariance [cerebrospinal fluid (CSF) signal: with CSFMask_3 mm; white matter signal: with WMMask_3 mm; head motion: Friston-24 parameters]; (i) temporal filtering $(0.01-0.1 \mathrm{~Hz}$ ); and (j) scrubbing (using default parameters according to the interpolation strategy: linear interpolation; FD threshold $=0.5$; previous time point number $=1$; subsequent time point number $=2$ ).

\section{Regions of Interest}

All ISSs occurred during the monitoring of motor reactions on the cortex. Hence, we focused on the sensorimotor network template that was extracted from a brain atlas, "brainnetome atlas" (http://www.brainnetome.org/) (22). The seeds were generated as spheres/circles of $5-\mathrm{mm}$ diameter based on the coordinates of the sensorimotor network. To avoid the effects of neurovascular uncoupling or tumor involvement, the regions invaded by gliomas were excluded (Supplementary Table 1).

\section{Network Construction}

To construct the functional connectivity (FC) matrix, Pearson's correlation coefficients were used to compare regional mean time series for all extracted nodes of sensorimotor networks.

\section{Graph Theoretical Measures}

Graph theoretical analyses were used to calculate global and nodal topological properties [the detailed information of topological properties is shown in the Supplementary Material (Part 1)]. All matrices were transformed into absolute and binary values to calculate topological properties.

\section{Statistical Analyses}

Clinical characteristics were compared between the patient groups using Student's $t$-test, Mann-Whitney $\mathrm{U}$ tests, chisquare tests, Fisher's exact tests, and one-way analysis of variance (ANOVA) according to the type of data. To explore group differences in network topological properties, we applied a series of sparsity thresholds (0.17-0.33; interval, 0.01) consistent with a previous study (23). False discovery rate (FDR) corrections were used to correct FC. Moreover, an etasquared correlation was applied to explore the relationship between ISS and FC values. Topological properties were compared among the groups by one-way ANOVA test. Least significant difference (LSD) was subsequently used for posthoc analysis when the results of one-way ANOVA were found
TABLE 1 | Demographic and clinical characteristics.

\begin{tabular}{|c|c|c|c|c|}
\hline $\begin{array}{l}\text { Demographic and } \\
\text { clinical characteristics }\end{array}$ & $\begin{array}{l}\text { ISS group } \\
(n=12)\end{array}$ & $\begin{array}{c}\text { Non-ISS } \\
\text { group }(n=15)\end{array}$ & $\begin{array}{l}\text { Healthy } \\
(n=20)\end{array}$ & $p$-value \\
\hline \multicolumn{5}{|l|}{ Gender } \\
\hline Male & 6 & 10 & 11 & 0.43 \\
\hline Female & 6 & 5 & 9 & \\
\hline Age $(y)^{\star}$ & $41.5 \pm 4.3$ & $44.4 \pm 3.0$ & $40.7 \pm 1.7$ & 0.57 \\
\hline \multicolumn{5}{|l|}{ Handedness } \\
\hline Right & 12 & 15 & 20 & - \\
\hline Left & 0 & 0 & 0 & \\
\hline \multicolumn{5}{|c|}{ KPS score (pre-operative) } \\
\hline 100 & 10 & 14 & - & 0.56 \\
\hline $90 \sim 100$ & 2 & 1 & - & \\
\hline \multicolumn{5}{|c|}{ KPS score (post-operative 3 months) } \\
\hline 100 & 10 & 15 & - & 0.18 \\
\hline $90 \sim 100$ & 2 & 0 & - & \\
\hline Education level (years) $^{\star}$ & $14.7 \pm 1.0$ & $15.2 \pm 0.9$ & $15.3 \pm 0.4$ & 0.85 \\
\hline \multicolumn{5}{|l|}{ Histopathology } \\
\hline Astrocytoma & 5 & 5 & - & - \\
\hline Oligodendroglioma & 3 & 4 & & \\
\hline Anaplastic astrocytoma & 1 & 3 & & \\
\hline Glioblastoma & 3 & 3 & & \\
\hline \multicolumn{5}{|l|}{ IDH status } \\
\hline Mutation & 6 & 6 & - & 0.78 \\
\hline Wild type & 6 & 9 & - & \\
\hline Tumor volume $(\mathrm{ml})^{\star}$ & $29.93 \pm 4.34$ & $33.98 \pm 5.29$ & - & 0.57 \\
\hline Stimulation current $(\mathrm{mA})^{\star}$ & $3.3 \pm 0.4$ & $3.0 \pm 0.3$ & - & 0.69 \\
\hline
\end{tabular}

*Values are means \pm standard error of the mean.

ISS group, group of patients with intraoperative stimulation-induced epilepsy.

Non-ISS group, group of patients without intraoperative stimulation-induced epilepsy. Using Mann-Whitney U-test to compare the difference of Karnofsky performance status between the ISS and non-ISS groups.

Using Student's t-test to compare the difference of tumor volume and stimulation current between the ISS and non-ISS groups.

Using one-way ANOVA test to compare the differences of age and education level between the ISS, non-ISS, and healthy groups.

Using Fisher's exact test to compare the differences of gender and locitrate dehydrogenase status between the ISS and non-ISS groups.

KPS, karnofsky performance status; IDH, iocitrate dehydrogenase.

to be significantly different between the three groups (the ISS, non-ISS, and healthy groups). A $p$-value $<0.05$ was considered significant.

\section{RESULTS}

\section{Demographic Characteristics}

Owing to the limited number of patients with right hemispheric gliomas accompanying ISS (two patients), 27 patients with left hemispheric gliomas were finally enrolled (men, $n=16$; all righthanded; Tables 1, 2). The screening process of recruiting patients is shown in Supplementary Figure 2. After matching for age, sex, and education level, 20 healthy participants were enrolled as controls (men, $n=11$ ).

No significant differences were observed in age, sex, or education level among the three groups. No differences were seen in preoperative and postoperative KPS scores, tumor 
TABLE 2 | Clinical information of enrolled patients.

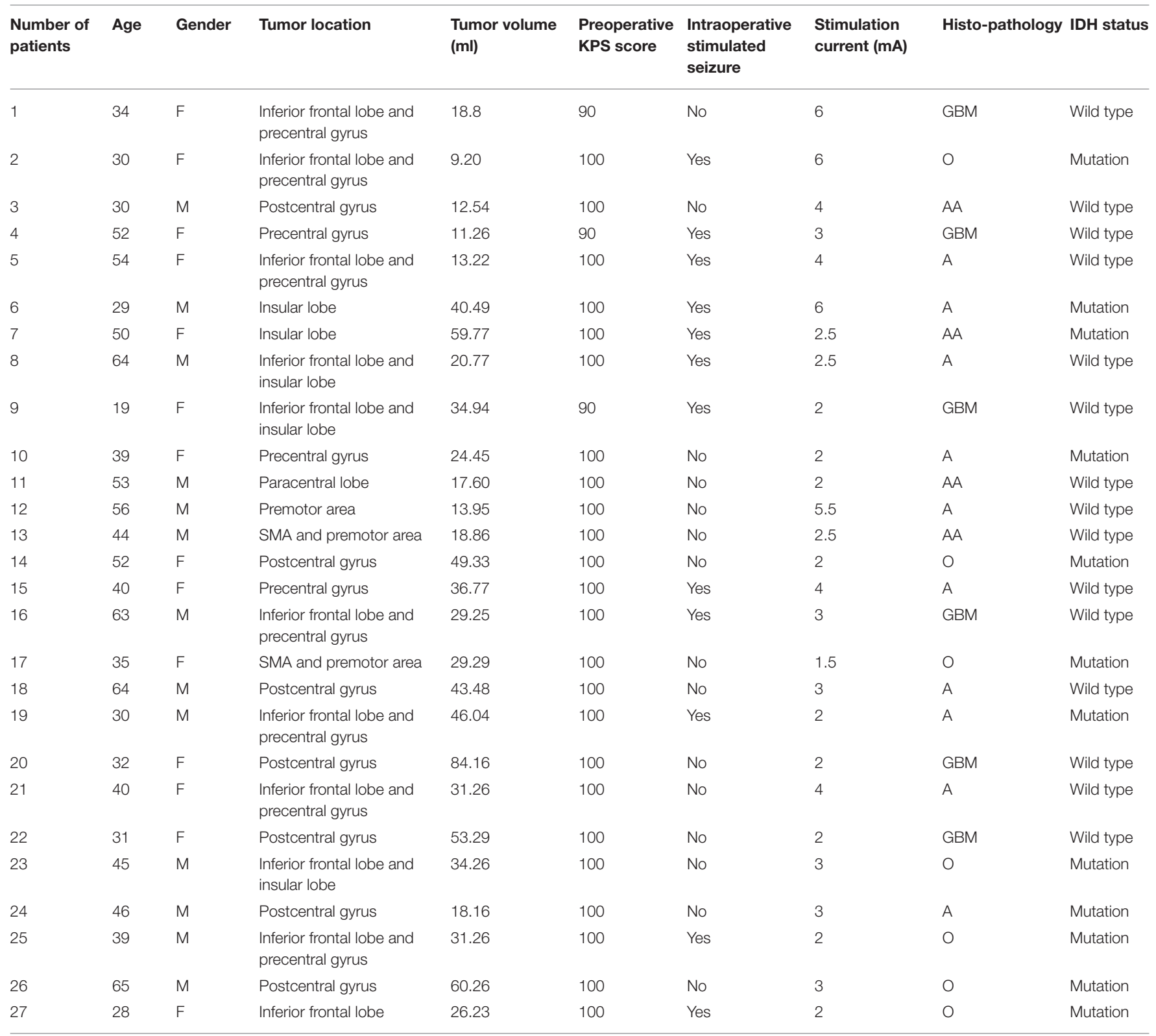

*KPS, karnofsky performance status; A, astrocytoma; O, oligodendroglioma; AA, anaplastic astrocytoma; GBM, glioblastoma; M, male; F, female.

volumes, stimulation current, and the proportion of patients with preoperative epilepsy between the ISS and non-ISS groups (Table 1).

\section{Functional Connectivity}

Compared with the non-ISS group, four functional edges had a decreased FC in the ISS and healthy groups after FDR correction ( $p$-value threshold $=0.002$; Figure 1, Supplementary Table 2) as follows: (1) the medial Brodmann area (BA) 6 in the right hemisphere (A6m_R) and BA 4 (head and face) in the right hemisphere (A4hf_R): ISS vs. non-ISS, $p<0.001$; non-ISS vs. healthy, $p<0.001$; (2) A4hf_R and the BA 4 (trunk) in the right hemisphere (A4t_R): ISS vs. non-ISS, $p<0.001$; non-ISS vs. healthy, $p<0.001$; (3) A4hf_R and the BA 4 (low limb) in the right hemisphere (A4ll_R): ISS vs. non-ISS, $p<0.001$; nonISS vs. healthy, $p<0.001$; and (4) the BA 4 (upper limb) in the right hemisphere (A4ul_R) and BA 1/2/3 (tongue and larynx) in the left hemisphere (A1_2_3tonIa_L): ISS vs. non-ISS, $p<0.001$; non-ISS vs. healthy, $p<0.001$.

Moreover, compared with the non-ISS group, the FC of the edge that was between BA 4 (upper limb) in the left hemisphere (A4ul_L) and BA 1/2/3 (tongue and larynx) in the left hemisphere (A1_2_3tonIa_L) only decreased in the ISS group (ISS vs. nonISS, $p<0.001)$.

Furthermore, compared with the non-ISS group, seven functional edges only decreased FC in the healthy group but were 




Differences between the Group ISS and Group non-ISS, simultanously between the Group non-ISS and Group Healthy

Differences between Group ISS and Group non-ISS

Differences between Group non-ISS and Group Healthy

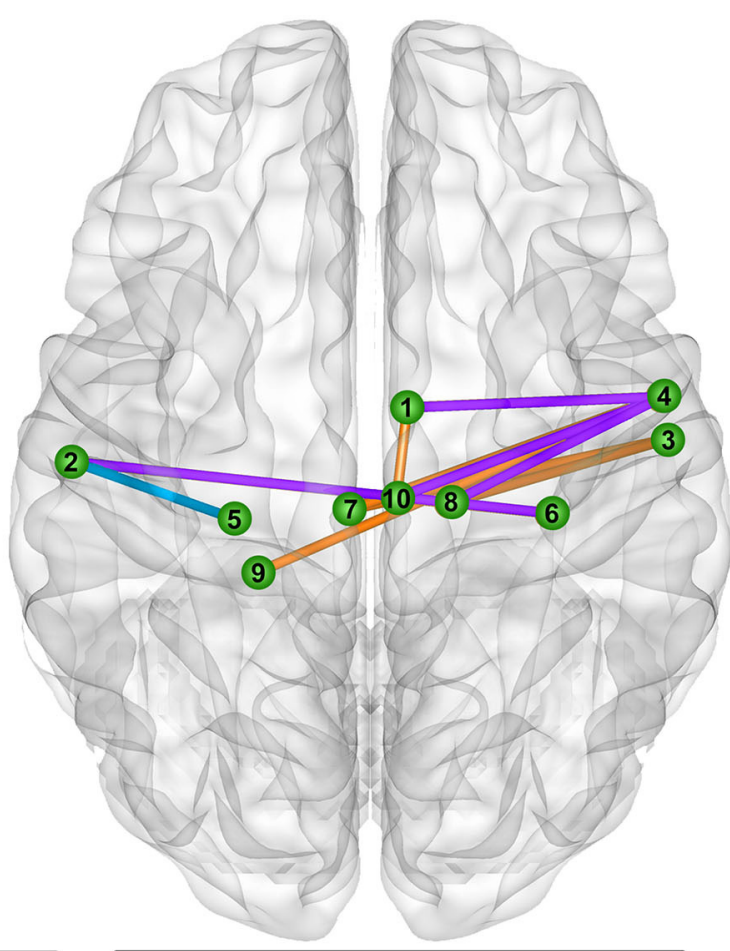

\begin{tabular}{|ll|}
\hline 1. A6m_R & 6. A4ul_R \\
2. A1/2/3tonla_L & 7. A4II_L \\
3. A1/2/3tonla_R & 8. A4t_R \\
4. A4hf_R & 9. A1/2/3tru_L \\
5. A4ul_L & 10. A4II_R \\
\hline
\end{tabular}

FIGURE 1 | Alterations in functional connectivity (FC) among the ISS, non-ISS, and healthy groups. ISS, group of patients with intraoperative stimulation-induced seizures; Non-ISS, group of patients without intraoperative stimulation-induced seizures.

insignificantly altered in the ISS group after FDR correction. The detailed information is shown in Supplementary Table 2.

\section{The Relationship Between Functional Connectivity and Intraoperative Stimulation-Induced Seizure Occurrence}

Four negative correlations were found between ISS occurrence and FC in four functional edges. The detailed correlations were as follows: (1) A6m_R and A4hf_R: $\mathrm{r}=-0.734, p<0.001$, etasquared correlation; (2) A4hf_R and A4t_R: $\mathrm{r}=-0.696, p=$ 0.001; (3) A4hf_R and A4ll_R: $r=-0.687, p=0.001$; and (4) A4ul_R and A1_2_3tonIa_L: $r=-0.695, p=0.001$.

\section{Global Topological Properties}

There were some differences in global efficiency $(p=0.041)$, shortest path length $(p=0.043)$, and local efficiency ( $p$ $=0.009$ ) between the three groups after one-way ANOVA (Supplementary Table 3, Figure 2).

On post-hoc analysis with LSD test, the non-ISS group $(0.636$ $\pm 0.004)$ showed weaker global efficiency than the ISS group $(0.651 \pm 0.002, p=0.027)$. Moreover, compared with the nonISS group $(1.966 \pm 0.034)$, the shortest path length was shorter in the ISS group $(1.850 \pm 0.013, p=0.026)$. Additionally, compared with the non-ISS group $(0.667 \pm 0.013)$, the local efficiency was greater in the healthy group $(0.740 \pm 0.011, p=0.002)$.

\section{Small Worldness Properties}

Our results showed that all three groups were a small-world network because gamma value was $>1$ and lambda was nearly equal to $1(\gamma>1, \lambda \approx 1$; Supplementary Table 3$)$. There were some differences observed in gamma value $(p=0.037)$ and sigma value $(p=0.046)$ among the three groups using one-way ANOVA (Supplementary Table 3, Figure 3).

After post-hoc analysis with LSD test, compared with the nonISS group $(1.075 \pm 0.030)$, the gamma value was greater in the healthy group $(1.249 \pm 0.038, p=0.011)$. Moreover, compared with the non-ISS group $(0.968 \pm 0.038)$, the sigma value was greater in the ISS $(1.127 \pm 0.059, p=0.044)$ and healthy $(1.14$ $\pm 0.041, p=0.017)$ groups.

\section{Nodal Topological Properties}

After one-way ANOVA, there were some differences in nodal efficiency between the three groups. These nodes were BA area 4 (tongue and larynx region) in the right hemisphere (A4tl_R, $p$ 

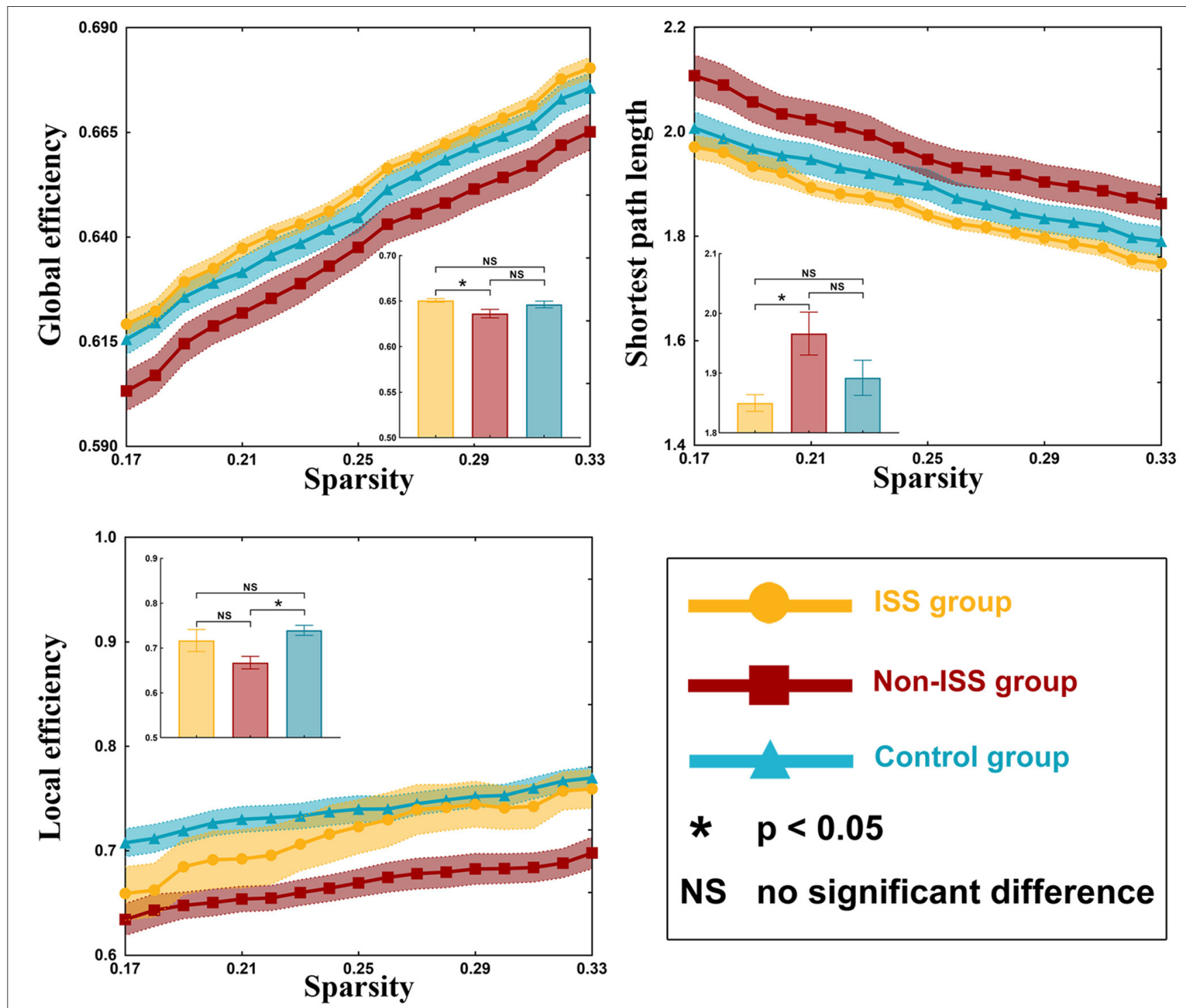

FIGURE 2 | Global properties of the ISS, non-ISS, and healthy groups. ISS, group of patients with intraoperative stimulation-induced seizures; Non-ISS, group of patients without intraoperative stimulation-induced seizures.

$=0.009$ ), $\mathrm{BA}$ area $1 / 2 / 3$ (lower limb region) in the left hemisphere $\left(\mathrm{A} 1 / 2 / 311 \_\mathrm{L}, p=0.022\right), \mathrm{BA}$ area 2 in the left hemisphere (A2_L, $p$ $=0.006)$, and A1/2/3tru_L ( $p=0.048)$ (Supplementary Table 4, Figure 4). No differences in other nodal properties (cluster efficiency, nodal shortest path length, and nodal local efficiency) were found among the three groups.

After post-hoc analysis with LSD test, compared with the non-ISS group $(0.182 \pm 0.071)$, the nodal efficiency of A4tl_R increased in the ISS $(0.466 \pm 0.054, p=0.007)$ and healthy $(0.437$ $\pm 0.052, p=0.006)$ groups. Moreover, compared with the nonISS group $(0.457 \pm 0.084)$, the nodal efficiency of A1/2/3ll_L increased in the healthy group (0.629 $\pm 0.018, p=0.007)$. Additionally, compared with the non-ISS group $(0.310 \pm 0.083)$, the nodal efficiency of A2_L increased in the ISS $(0.563 \pm 0.067$, $p=0.006)$ and healthy $(0.549 \pm 0.025, p=0.007)$ groups.
Furthermore, compared with the non-ISS group (0.572 \pm 0.064$)$, the nodal efficiency of A1/2/3tru_L increased in the ISS (0.692 \pm $0.015, p=0.028)$ and healthy $(0.675 \pm 0.014, p=0.029)$ groups.

\section{DISCUSSION}

This study investigated the alterations of FC and topological properties in the sensorimotor networks of patients with and without ISS during DCS. We found that the glioma induced some totally opposing alterations in FC and topological properties, thus leading to different susceptibilities to ISS in patients.

In our treatment center, ISS incidence during AC was $16.7 \%$ $(14 / 84)$ using the same routine stimulation protocol as in a previous study (24). This was consistent with that reported in previous studies $(2.2-21.5 \%)(1,4-14)$. 

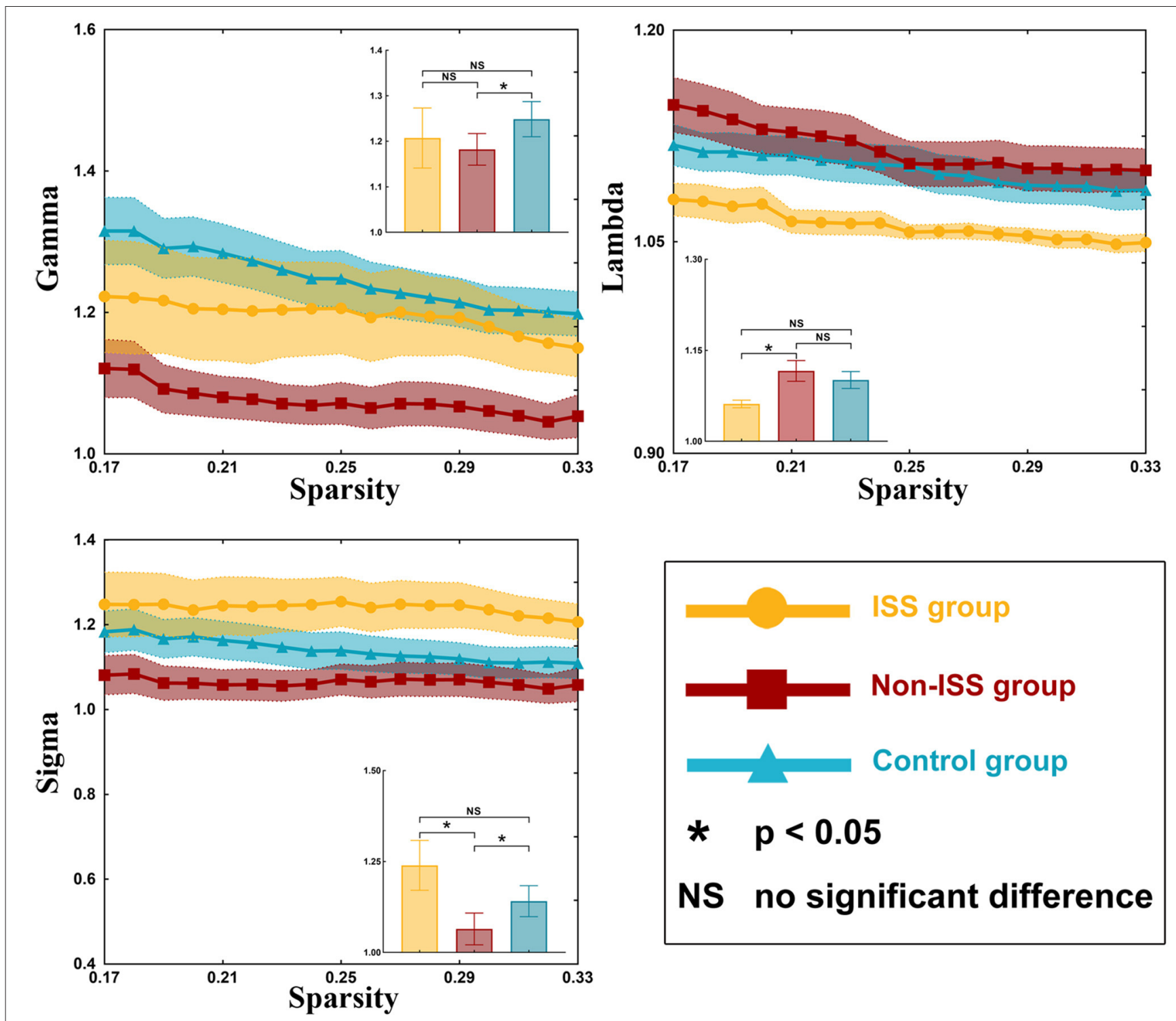

FIGURE 3 | Small-world properties of the ISS, non-ISS, and healthy groups. ISS, group of patients with intraoperative stimulation-induced seizures; Non-ISS, group of patients without intraoperative stimulation-induced seizures.

In this study, all ISSs occurred during sensory and motor function monitoring. All sites inducing ISS were located in the sensorimotor network. Consequently, we mainly focused on alterations in the sensorimotor network.

\section{Functional Connectivity Alterations}

Different alterations in FC were associated with ISS. Here, we found that there were four edges that decreased FC in the ISS group and simultaneously increased FC in the non-ISS group. It is widely accepted that glioma can disrupt functional networks and induce reorganization of the disrupted networks $(25,26)$. In our study, no patient had preoperative motor or sensory findings, indicating that sensorimotor functions of these patients were compensated. Hence, all FC alterations in the sensorimotor network were related to glioma-induced disruption and reorganization. Additionally, our findings showed that the glioma-induced reorganization was different. Such difference may lead to the stratification of susceptibility to ISS. Furthermore, our findings regarding the negative correlations between FC and occurrence of ISS revealed that the decreased FC may be a potential marker for identifying patients susceptible to ISS.

\section{Topological Property Alterations}

Glioma-induced alterations of topological properties in ISS patients were converse to those of patients without ISS. The increased global efficiency, sigma value, and decreased shortest path length represented that the ability of information transiting 


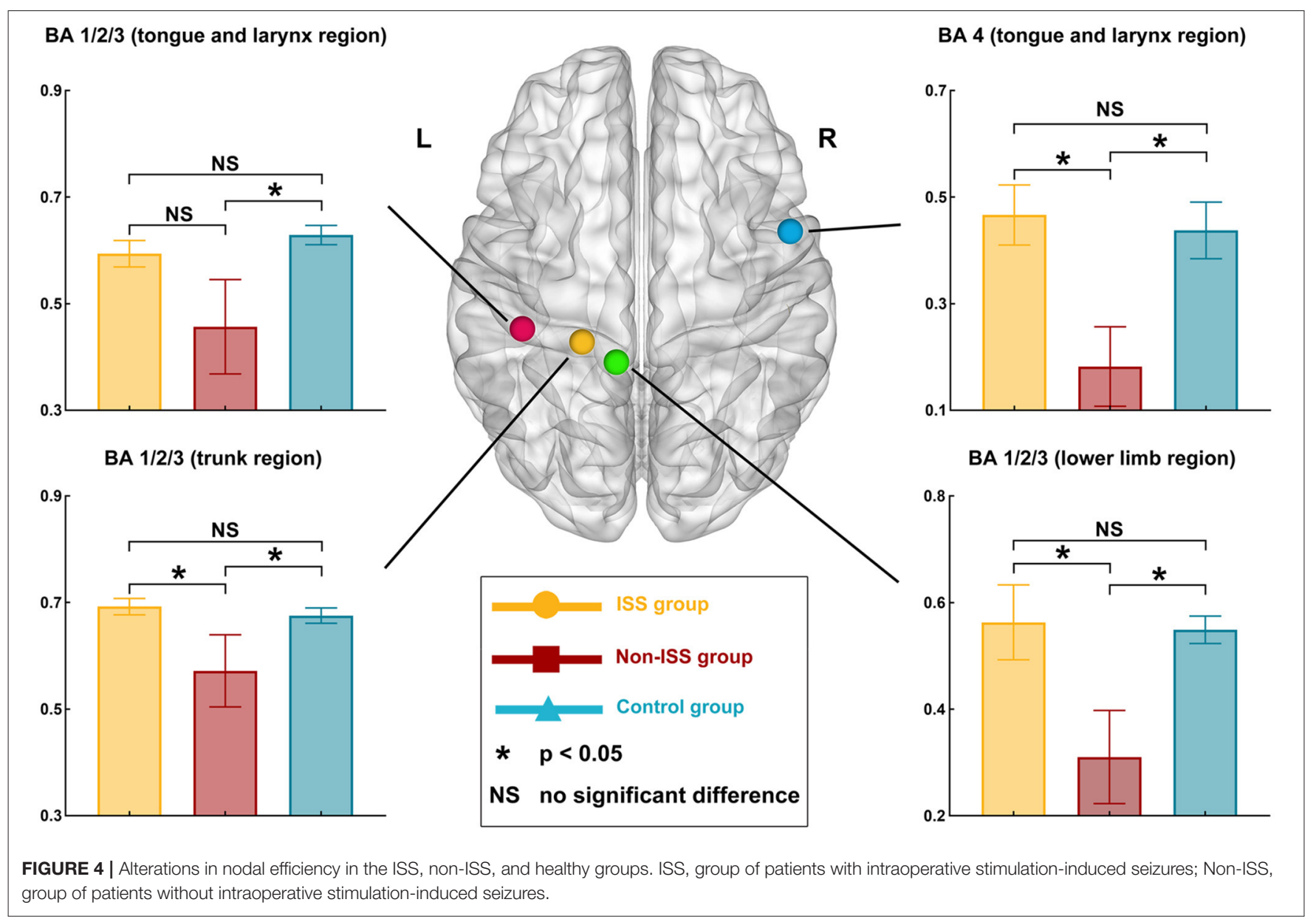

is strong. Hence, these results indicated that gliomas could strengthen the ability of information conduction in ISS patients and weaken them in non-ISS patients. Moreover, a shortening network path contributes to reducing the convulsive threshold underlying epileptic seizures $(27,28)$. Conversely, longer network paths are related to a prolonged system response time that counteracts the rapid spread of local epileptic discharges (29). Consequently, the strengthened ability of information conduction and shortened network pathway can make the patients susceptible to ISS. Some studies focused on the temporal lobe epilepsy found that the longer shortest path length was related to seizure onset (30). We thought that this difference was due to different pathologies between idiopathic seizure and glioma-related epilepsy. The low-grade glioma grows slowly and induces network reorganization easily, and the idiopathic seizure often leads to gray matter atrophy (31) and hypometabolism (32). Hence, the decreased shortest path length was often found in patients with glioma-related epilepsy, and the increased shortest path length was often found in patients with temporal lobe epilepsy (20). Moreover, our results showed that nodal efficiency of three regions of BA $1 / 2 / 3$ areas (including trunk, tongue, and lower limb) increased in the ISS group. These findings indicated that those nodes were activated to participate in the gliomainduced motion generation process in the ISS group, but those nodes were inhibited from participating in this process in the non-ISS group. The BA $1 / 2 / 3$ areas were directly associated with information conduction of motion generation and control $(33,34)$. Hence, we believe that the increased ability of information conduction induced by glioma specifically implied patients with glioma would develop ISS during DCS. In addition, no differences in preoperative epileptic status and stimulation current were found between the ISS and nonISS groups. Thus, we believe that the specific alterations in the sensorimotor network were more likely to be induced by glioma itself.

\section{Value of the Current Study}

Duffau et al. (25) proposed that using ECoG was unnecessary. Because the low-intensity stimulation could not result in ISS, the absence of ECoG monitoring could simplify the surgical procedure. However, under the same stimulation protocol without ECoG monitoring, our patients experienced ISS [incidence was four times of that reported by Duffau (25)] with 1 or $1.5 \mathrm{~mA}$ as the initial stimulation current. Hence, we recommend that ECoG should not be omitted in some patients. Fortunately, the specific glioma-induced alterations in patients susceptible to ISS were found. These findings indicated that preoperative rs-fMRI may contribute to identifying patients who are more susceptible to ISS. 
For these susceptible patients, neurosurgeons should use ECoG to capture after-discharge current during DCS to avoid ISS.

\section{CONCLUSION}

Patients with increased global and nodal efficiency, decreased shortest path length, and decreased FC induced by glioma are susceptible to ISS during DCS. In such patients, ECoG is recommended to monitor after-discharges during DCS to prevent ISS.

\section{DATA AVAILABILITY STATEMENT}

Anonymized data will be made available on request.

\section{ETHICS STATEMENT}

The studies involving human participants were reviewed and approved by IRB of Beijing Tiantan Hospital. Written informed consent to participate in this study was provided by the participants' legal guardian/next of kin.

\section{AUTHOR CONTRIBUTIONS}

SF and CZ contributed to the study concept and design. SF, XF, $\mathrm{YW}$, and ZZ contributed to data acquisition and analysis. SF, LW,

\section{REFERENCES}

1. Sacko O, Lauwers-Cances V, Brauge D, Sesay M, Brenner A, Roux FE. Awake craniotomy vs surgery under general anesthesia for resection of supratentorial lesions. Neurosurgery. (2011) 68:1192-8; discussion 11989. doi: 10.1227/NEU.0b013e31820c02a3

2. Hervey-Jumper SL, Li J, Lau D, Molinaro AM, Perry DW, Meng L, et al. Awake craniotomy to maximize glioma resection: methods and technical nuances over a 27-year period. J. Neurosurg. (2015) 123:32539. doi: 10.3171/2014.10.JNS141520

3. Sartorius CJ, Berger MS. Rapid termination of intraoperative stimulation-evoked seizures with application of cold Ringer's lactate to the cortex. Technical note. J. Neurosurg. (1998) 88:349-51. doi: 10.3171/jns.1998.88.2.0349

4. Nossek E, Matot I, Shahar T, Barzilai O, Rapoport Y, Gonen T, et al. Failed awake craniotomy: a retrospective analysis in 424 patients undergoing craniotomy for brain tumor. J. Neurosurg. (2013) 118:2439. doi: $10.3171 / 2012.10$.JNS12511

5. Grossman R, Nossek E, Sitt R, Hayat D, Shahar T, Barzilai O, et al. Outcome of elderly patients undergoing awake-craniotomy for tumor resection. Ann. Surg. Oncol. (2013) 20:1722-8. doi: 10.1245/s10434-0122748-x

6. Conte V, Magni L, Songa V, Tomaselli P, Ghisoni L, Magnoni $\mathrm{S}$, et al. Analysis of propofol/remifentanil infusion protocol for tumor surgery with intraoperative brain mapping. J. Neurosurg. Anesthesiol. (2010) 22:119-27. doi: 10.1097/ANA.0b013e3181c $959 f 4$

7. Pereira LC, Oliveira KM, L'Abbate GL, Sugai R, Ferreira JA, da Motta LA. Outcome of fully awake craniotomy for lesions near the eloquent cortex: analysis of a prospective surgical series of 79 supratentorial primary brain tumors with long follow-up. Acta Neurochir. (2009) 151:121530. doi: 10.1007/s00701-009-0363-9
$\mathrm{ZZ}$, and YW contributed to the statistics/verified the analytical method. SF, CZ, and YW contributed to writing the first draft. $\mathrm{XF}, \mathrm{ZZ}, \mathrm{YW}$, and TJ supervised the study, read and approved the final version. All authors contributed to the article and approved the submitted version.

\section{FUNDING}

This work was supported by the Public Welfare Development and Reform Pilot Project of Beijing Medical Research Institute (No. PXM2019_026280_000008), Beijing Municipal Natural Science Foundation (No. 7202021), National Natural Science Foundation of China (No. 82001777), and Research Unit of Accurate Diagnosis, Treatment, and Translational Medicine of Brain Tumors Chinese (No. 2019-I2M-5-021).

\section{ACKNOWLEDGMENTS}

We would like to thank Dr. Lanxi Meng for imaging data acquisition for this study.

\section{SUPPLEMENTARY MATERIAL}

The Supplementary Material for this article can be found online at: https://www.frontiersin.org/articles/10.3389/fneur. 2021.602716/full\#supplementary-material

8. Serletis D, Bernstein M. Prospective study of awake craniotomy used routinely and nonselectively for supratentorial tumors. J. Neurosurg. (2007) 107:16. doi: 10.3171/JNS-07/07/0001

9. Skucas P, Artru AA. Anesthetic complications of awake craniotomies for epilepsy surgery. Anesth. Analg. (2006) 102:8827. doi: 10.1213/01.ane.0000196721.49780.85

10. Sarang A, Dinsmore J. Anaesthesia for awake craniotomy-evolution of a technique that facilitates awake neurological testing. Br. J. Anaesth. (2003) 90:161-5. doi: 10.1093/bja/aeg037

11. Herrick A, Craen RA, Gelb AW, Miller LA, Kubu CS, Girvin JP, et al. Propofol sedation during awake craniotomy for seizures: patient-controlled administration versus neurolept analgesia. Anesth. Analg. (1997) 84:128591. doi: 10.1213/00000539-199706000-00021

12. Herrick A, Craen RA, Gelb AW, McLachlan RS, Girvin JP, Parrent AG, et al. Propofol sedation during awake craniotomy for seizures: electrocorticographic and epileptogenic effects. Anesth. Analg. (1997) 84:1280-4. doi: 10.1213/00000539-199706000-00020

13. Gignac E, Manninen PH, Gelb AW. Comparison of fentanyl, sufentanil and alfentanil during awake craniotomy for epilepsy. Can. J. Anaesth. (1993) 40(5 Pt 1):421-4. doi: 10.1007/BF03009510

14. Archer DP, McKenna JM, Morin L, Ravussin P. Conscious-sedation analgesia during craniotomy for intractable epilepsy: a review of 354 consecutive cases. Can. J. Anaesth. (1988) 35:338-44. doi: 10.1007/BF03010852

15. Nossek E, Matot I, Shahar T, Barzilai O, Rapoport Y, Gonen T, et al. Intraoperative seizures during awake craniotomy: incidence and consequences: analysis of 477 patients. Neurosurgery. (2013) 73:135-40; discussion 140. doi: 10.1227/01.neu.0000429847.91707.97

16. Boetto J, Bertram L, Moulinie G, Herbet G, Moritz-Gasser S, Duffau H. Low rate of intraoperative seizures during awake craniotomy in a prospective cohort with 374 supratentorial brain lesions: electrocorticography is not mandatory. World Neurosurg. (2015) 84:1838-44. doi: 10.1016/j.wneu.2015.07.075 
17. Fang S, Liang J, Qian T, Wang Y, Liu X, Fan X, et al. Anatomic location of tumor predicts the accuracy of motor function localization in diffuse lowergrade gliomas involving the hand knob area. AJNR Am. J. Neuroradiol. (2017) 38:1990-7. doi: 10.3174/ajnr.A5342

18. Wang J, Wang X, Xia M, Liao X, Evans A, He Y. Corrigendum: GRETNA: a graph theoretical network analysis toolbox for imaging connectomics. Front. Hum. Neurosci. (2015) 9:458. doi: 10.3389/fnhum.2015. 00458

19. Wang J, Wang X, Xia M, Liao X, A. Evans, He Y. GRETNA: a graph theoretical network analysis toolbox for imaging connectomics. Front. Hum. Neurosci. (2015) 9:386. doi: 10.3389/fnhum.2015.00386

20. Fang S, Zhou C, Fan X, Jiang T, Wang Y. Epilepsy-related brain network alterations in patients with temporal lobe glioma in the left hemisphere. Front. Neurol. (2020) 11:684. doi: 10.3389/fneur.2020.00684

21. Calhoun VD, Wager TD, Krishnan A, Rosch KS, Seymour KE, Nebel MB, et al. The impact of T1 versus EPI spatial normalization templates for fMRI data analyses. Hum. Brain Mapp. (2017) 38:5331-42. doi: 10.1002/hbm. 23737

22. Fan L, Li H, Zhuo J, Zhang Y, Wang J, Chen L, et al. The human brainnetome atlas: a new brain atlas based on connectional architecture. Cereb. Cortex. (2016) 26:3508-26. doi: 10.1093/cercor/bhw157

23. Ji GJ, Yu Y, Miao HH, Wang ZJ, Tang YL, Liao W. Decreased network efficiency in benign epilepsy with centrotemporal spikes. Radiology. (2017) 283:186-94. doi: 10.1148/radiol.2016160422

24. Fang S, Li Y, Wang Y, Zhang Z, Jiang T. Awake craniotomy for gliomas involving motor-related areas: classification and function recovery. J. Neurooncol. (2020) 148:317-25. doi: 10.1007/s11060-020-0 3520-w

25. Duffau H. The "frontal syndrome" revisited: lessons from electrostimulation mapping studies. Cortex. (2012) 48:120-31. doi: 10.1016/j.cortex.2011.04.029

26. Herbet G, Maheu M, Costi E, Lafargue G, Duffau H. Mapping neuroplastic potential in brain-damaged patients. Brain. (2016) 139(Pt 3):829-44. doi: 10.1093/brain/awv394

27. Ponten SC, Bartolomei F, Stam CJ. Small-world networks and epilepsy: graph theoretical analysis of intracerebrally recorded mesial temporal lobe seizures. Clin. Neurophysiol. (2007) 118:918-27. doi: 10.1016/j.clinph.2006.12.002
28. Dyhrfjeld-Johnsen J, Santhakumar V, Morgan RJ, Huerta R, Tsimring L, Soltesz I. Topological determinants of epileptogenesis in large-scale structural and functional models of the dentate gyrus derived from experimental data. $J$. Neurophysiol. (2007) 97:1566-87. doi: 10.1152/jn.00950.2006

29. Ofer I, LeRose C, Mast H, LeVan P, Metternich B, Egger K, et al. Association between seizure freedom and default mode network reorganization in patients with unilateral temporal lobe epilepsy. Epilepsy Behav. (2019) 90:23846. doi: 10.1016/j.yebeh.2018.10.025

30. Haneef Z, Chiang S. Clinical correlates of graph theory findings in temporal lobe epilepsy. Seizure. (2014) 23:809-18. doi: 10.1016/j.seizure.2014.07.004

31. Caciagli L, Bernasconi A, Wiebe S, Koepp MJ, Bernasconi $\mathrm{N}$, Bernhardt BC. A meta-analysis on progressive atrophy in intractable temporal lobe epilepsy: time is brain? Neurology. (2017) 89:506-16. doi: 10.1212/WNL.0000000000004176

32. Celiker Uslu S, Yuksel B, Tekin B, Sariahmetoglu H, Atakli D. Cognitive impairment and drug responsiveness in mesial temporal lobe epilepsy. Epilepsy Behav. (2019) 90:162-7. doi: 10.1016/j.yebeh.2018.10.034

33. Klingner M, Brodoehl S, Witte OW, Guntinas-Lichius O, Volk GF. The impact of motor impairment on the processing of sensory information. Behav. Brain Res. (2019) 359:701-8. doi: 10.1016/j.bbr.2018.09.016

34. Borich R, Brodie SM, Gray WA, Ionta S, Boyd LA. Understanding the role of the primary somatosensory cortex: opportunities $\begin{array}{llll}\text { for } & \text { rehabilitation. Neuropsychologia. (2015) } & 79(\mathrm{Pt} & \mathrm{B}): 246-\end{array}$ 55. doi: 10.1016/j.neuropsychologia.2015.07.007

Conflict of Interest: The authors declare that the research was conducted in the absence of any commercial or financial relationships that could be construed as a potential conflict of interest.

Copyright (c) 2021 Fang, Zhou, Wang, Fan, Wang, Zhang and Jiang. This is an open-access article distributed under the terms of the Creative Commons Attribution License (CC BY). The use, distribution or reproduction in other forums is permitted, provided the original author(s) and the copyright owner(s) are credited and that the original publication in this journal is cited, in accordance with accepted academic practice. No use, distribution or reproduction is permitted which does not comply with these terms. 